\title{
A luminosity model of RHIC gold runs
}

\author{
S.Y.Zhang
}

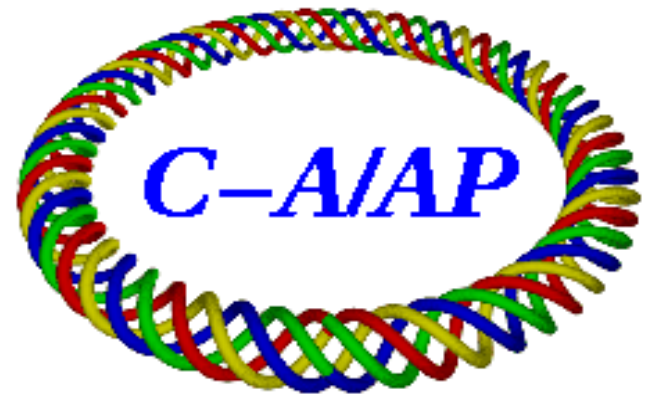

\section{Collider-Accelerator Department Brookhaven National Laboratory \\ Upton, NY 11973}

Notice: This document has been authorized by employees of Brookhaven Science Associates, LLCunder Contract No. DE-AC02-98CH10886 with the U.S. Departm ent of En ergy. The United States Governm ent retains a nonexclusive, paid-up, irrevocable, world-wide license topublish or reproduce the published formof this document, or allow others to do so, for United States Government purposes. 


\title{
A Luminosity Model of RHIC Gold Runs *
}

\author{
S.Y. Zhang
}

\begin{abstract}
In this note, we present a luminosity model for RHIC gold runs. The model is applied to the physics fills in 2007 run without cooling, and with the longitudinal cooling applied to one beam only. Having good comparison, the model is used to project a fill with the longitudinal cooling applied to both beams. Further development and possible applications of the model are discussed.
\end{abstract}

\section{Introduction}

To maximize the integrated luminosity, usually the higher beam intensity, smaller longitudinal and transverse emittance, and smaller $\beta^{*}$ are the directions to work on. In past 10 years, the RHIC gold runs have demonstrated a path toward this goal [1]. Most recently, a successful commissioning of the bunched beam stochastic cooling, both longitudinal and transverse, has offered a chance of further RHIC luminosity improvement $[2,3]$. With so many factors involved, a luminosity model would be useful to identify and project gains in the machine development. In this article, a preliminary model is proposed.

In Section 2, several secondary factors, which are not yet included in the model, are identified based on the RHIC operation condition and experience in current runs.

In Section 3, the RHIC beam store parameters used in the model are listed, and validated.

In Section 4, the factors included in the model are discussed, and the luminosity model is presented.

In Section 5, typical RHIC gold fills without cooling, and with partial cooling are used for comparison with the model. Then a projection of fills with more coolings is shown.

In Section 6, further development of the model is discussed.

*This note was finished March 2011, the development and applications regarding RHIC Au Run2011 is not included, and will be reported in future. 


\section{Secondary factors}

Based on the current RHIC operations, several secondary factors of the luminosity are discussed. These factors are not included in the model, but further machine improvement may necessitate the inclusion of some factors, for example, the beambeam and the dynamic aperture.

\section{$2.1 \quad$ Electron cloud}

For RHIC gold runs, the electron cloud is peaked at the transition, and it is reduced significantly at store. We take Fill 11858 in Run 2010 as an example. The injected beam intensity is $150 \times 10^{9}$ ions in both rings. Among 12 long warm straight sections for Yellow beam, only Yo4 and Yi11 are at $10^{-9}$ Torr, all others are below $10^{-10}$ Torr. For Blue, only Bo3 and Bi4 are at $10^{-9}$ Torr, others are below $10^{-10}$ Torr. Taking average pressure rise in rings as $2 \times 10^{-10}$ Torr, and assuming half of that is due to the electron cloud, with the desorption rate of $\eta_{e}=0.005$, then the average electron density in rings is about $10^{8} \mathrm{~m}^{-3}$. With this electron density, the effect on the beam emittance is not significant [4].

\subsection{Beam-gas collision}

At RHIC store, the capture cross section of gold ions is about $0.11 \times 10^{-24} \mathrm{~cm}^{2}$, but the nuclear collision cross section is much larger, at $4.65 \times 10^{-24} \mathrm{~cm}^{2}[5,6]$. We use the following equation to estimate the beam intensity life time,

$$
\frac{N}{N_{0}}=\exp \int_{0}^{t}-\sigma_{\text {total }} n d \ell
$$

where $\sigma_{\text {total }}$ is the total cross section, $n=3.3 \times 10^{16} P$ is the number of moleculae per $\mathrm{cm}^{3}$ at the pressure of $P$ Torr, and $\ell=\beta c$ is the path of projectile.

Taking vacuum pressure of $2 \times 10^{-10}$ Torr, and considering that the warm sections is about $800 \mathrm{~m}$ with the RHIC circumference of $3834 \mathrm{~m}$, then the beam life time due to the beam-gas collision is calculated as 1450 hours.

\subsection{Beam-beam}

The beam-beam parameter is defined as

$$
\xi=\frac{M_{i p} N_{b h} r_{i}}{4 \pi \epsilon_{N, r m s}}
$$

where $M_{i p}$ is the number of collision point, $N_{b h}$ is the bunch intensity, $r_{i}$ is the classical radius of ions, and $\epsilon_{N, r m s}$ is the normalized rms transverse emittance. With 2 collision points, $10^{9}$ ions per bunch, and $\epsilon_{N, r m s}=2.5 \pi \mu m$, the total beam-beam parameter is $\xi=0.003$. We note, however, that either with more experiments, and/or 
higher bunch intensity, and/or smaller emittance, the beam-beam may become im-

portant. For example, doubling the bunch intensity, and reducing the emittance by factor of 2, which has been demonstrated in recent run by applying vertical cooling, would bring the beam-beam parameter to $\xi=0.012$. This beam-beam parameter has been reached in RHIC proton runs, and the associated transverse emittance growth becomes an important factor in the integrated luminosity [7].

\subsection{Radiation damping}

With the RHIC bending field and beam energy at store, the radiation damping has no significant effect on the beam emittance. For example, the longitudinal and transverse damping time due to the radiation mechanism is 825 hour and 1650 hour, respectively. This is compared with LHC at store, 6.3 hour and 12.6 hour, respectively [8].

\subsection{Dynamic aperture}

With the squeezing $\beta^{*}$, the dynamic aperture becomes a main focus in recent RHIC gold run of $2010[9,10]$. The $\beta^{*}$ is not the only issue. Other issues related to the dynamic aperture include,

- With same $\beta^{*}$, different lattice design could lead to different dynamic aperture.

- From the machine operations, the choice of the working point and various non-linearity corrections might play important roles.

- The beam momentum spread is a crucial parameter in defining the dynamic aperture, therefore, the beam longitudinal emittance and the RF voltage applied also make big difference.

The effect of the dynamic aperture is mainly on the beam loss, and this effect will be gradually included in the model, following the RHIC machine development.

\section{RHIC beam parameter at store}

Typical RHIC gold beam store parameters are shown in Table 1, which is similar to the ones achieved in RHIC, for example, the Golden fill in 2010, 11858. The beam parameters are at the initial beam collision. 


\begin{tabular}{ccc}
\hline \hline Parameter & Unit & RHIC collision \\
\hline Energy, $E$ & $G e V / n$ & 100 \\
Betastar, $\beta^{*}$ & $m$ & 0.7 \\
Bunch number, $M$ & & 110 \\
Bunch intensity, $N_{y}, N_{b}$ & $10^{9}$ & 1.15 \\
Normalized transverse emittance, rms, $\epsilon_{N, r m s}$ & $\pi \mu m$ & 2.5 \\
Longitudinal emittance, $\epsilon_{\ell, 95 \%}$ & $e V s / n$ & 0.7 \\
Momentum spread, rms, $\delta_{p}$ & $10^{-3}$ & 0.67 \\
RF voltage, $V$ & $M V$ & 4 \\
RF harmonic, $h$ & & 2520 \\
Bunch length, rms, $\sigma_{s}$ & $m$ & 0.3 \\
Number of IP, $M_{i p}$ & & 2 \\
Peak luminosity, $\mathcal{L}_{\text {peak }}$ & $10^{27} \mathrm{~cm}^{-2} s^{-1}$ & 3.6 \\
beam-beam parameter, $\xi$ & $10^{-3}$ & 3.5 \\
Luminosity lifetime, $\tau_{\text {lumi }}$ & $h o u r$ & 5.5 \\
\hline \hline
\end{tabular}

Table 1: RHIC gold beam store parameters. The beam intensity, emittance, bunch length, and luminosity are at the initial collision.

If the luminosity model is applied to a specific fill, some adjustment has to be made. For example, in entire run 2007, $\beta^{*}=0.8 m$ and bunch number is $M=103$, which are different from Table 1 . Also, the initial bunch intensity in store is different from fill to fill, depending on the operation.

Several fundamental parameters, however, have not changed drastically from fill to fill, or even from run to run. After all, if not so, one may wonder the universal value of a luminosity model. These parameters include the transverse emittance, the longitudinal emittance, the beam momentum spread, and the bunch length, all at the initial collision. In the following, these parameters will be validated according to the RHIC current operations.

\subsection{Transverse emittance}

The transverse emittance is monitored at RHIC by the luminosity and the beam intensity from the DC transformer. Once the machine is well tuned, usually at the later part of a run, the transverse emittance at the start of the collision is not changed too much from fill to fill, even from run to run.

In Table 2, the normalized rms transverse emittance at the beginning of the collision are shown for the latest 50 fills in 2007 and in 2010, respectively. The bunch intensities of these fills are also shown. 

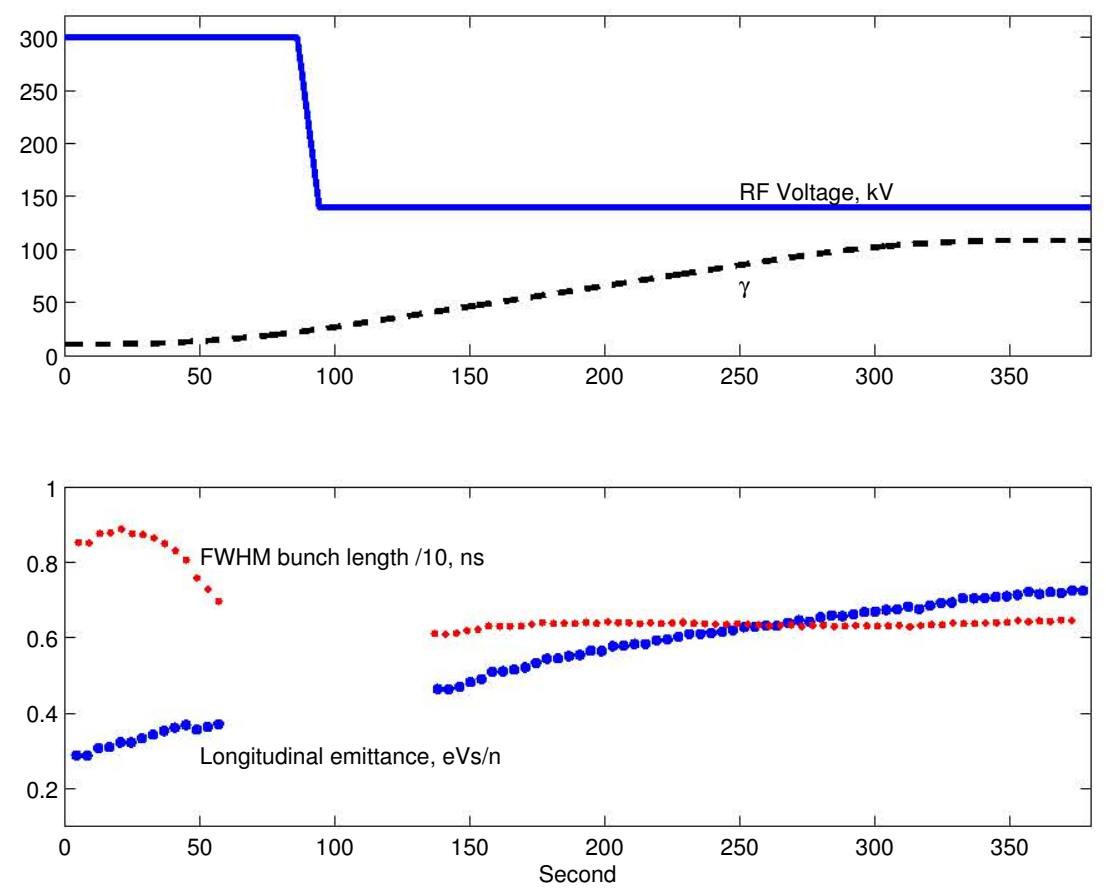

Figure 1: Beam longitudinal emittance during the acceleration, calculated from the FWHM bunch length measured by WCM. At the injection the emittance is 0.3 $e V s / n$, and at the full energy it is about $0.7 \mathrm{eVs} / n$. The RF voltage and the $\gamma$ function are also shown. The transition is at about 90 second in the plot.

\begin{tabular}{ccccc}
\hline \hline Parameter & \multicolumn{2}{c}{2007} & \multicolumn{2}{c}{2010} \\
& \multicolumn{2}{c}{ Fill $8901-9056$} & \multicolumn{2}{c}{ Fill $11772-11888$} \\
\hline & Average & Std. deviation & Average & Std. deviation \\
\hline Bunch intensity, $10^{9}$ & 1.050 & 0.061 & 1.096 & 0.062 \\
Norm. rms emittance, $\pi \mu m$ & 3.155 & 0.232 & 3.152 & 0.419 \\
\hline \hline
\end{tabular}

Table 2: Bunch intensity and transverse emittance at the start of the collision, for the latest 50 fills in 2007 and 2010, respectively.

The transverse emittance calculated this way, however, is larger than it should be. The hour glass factor and other corrections are not included. The bunched beam intensity, which is responsible for the luminosity production, is smaller than the beam intensity measured from the DC transformer. Therefore, $\epsilon_{N, r m s}=2.5$ $\pi \mu m$ is used for the initial value in the model.

\subsection{Longitudinal emittance}

In both run 2007 and run 2010, the beam injected into RHIC with the longitudinal emittance of $\epsilon_{\ell, 95 \%} \approx 0.3 \mathrm{eVs} / \mathrm{n}$. There are, however, a large growth during 
the acceleration, mainly due to the intrabeam scattering, and sometimes it is also accompanied by a large growth at the transition.

In Fig.1, using the FWHM (full width half magnitude) bunch length measured from the WCM (wall current monitor) of Fill 11277 in run 2010, the longitudinal emittance growth during the acceleration is shown. The bunch length measurement is usually absent around the transition, which is at about 90 second from the start of the acceleration. The RF voltage of the $28 \mathrm{MHz}$ cavities, which is used for acceleration, is also shown.

At the flatop, with the energy of $100 \mathrm{GeV} / n$ and $\gamma=107$, the longitudinal emittance is settled at $\epsilon_{\ell, 95 \%} \approx 0.7 \mathrm{eVs} / \mathrm{n}$.

\subsection{Momentum spread}

Before the collision, the beam is re-captured (rebucketing) by the $197 \mathrm{M} \mathrm{Hz}$ cavities. The beam momentum spread at the early store is determined by not only the longitudinal emittance, but also the RF voltage. With $4 \mathrm{MV}$, the $197 \mathrm{MHZ}$ cavity bucket area is smaller than $1 \mathrm{eVs} / n$, close to the beam emittance. The $95 \%$ beam

momentum spread is taken as $(d p / p)_{95 \%}=0.00134$, which is slightly smaller than the bucket half height of 0.00149 with the RF voltage of $4 M V$.

The rms momentum spread is a half of the $95 \%$ momentum spread, i.e.,

$$
(d p / p)_{95 \%}=2 \delta_{p}
$$

therefore, the rms beam momentum spread is taken as $\delta_{p}=0.00067$.

\subsection{Bunch length}

With the rebucketing of $197 \mathrm{MHz}$ cavities, the center bucket is always filled up. The bunch line density of the particles in the center bucket is Gaussian. In Fig.2, the beam line density of Fill 8825 in run 2007 is compared with a Gaussian of the rms bunch length $1 \mathrm{~ns}$, or $\sigma_{s}=0.3 \mathrm{~m}$.

Fill 8825, like some other fills, has a longitudinal emittance a little larger than $0.7 \mathrm{eVs} / \mathrm{n}$ at the rebucketing, and the $\mathrm{RF}$ voltage is about $3 \mathrm{MV}$. Some particles are captured by the adjacent side buckets. This is shown as the side lobes of the beam line density in Fig.2.

Therefore, we take the rms bunch length to be $0.3 \mathrm{~m}$, and keep in mind that the effect of side lobes is not negligible.

\section{Luminosity model}

The factors regarding the beam intensity, the emittance, and directly the luminosity, and hence included in the luminosity model are discussed in this section. The model is then presented. 


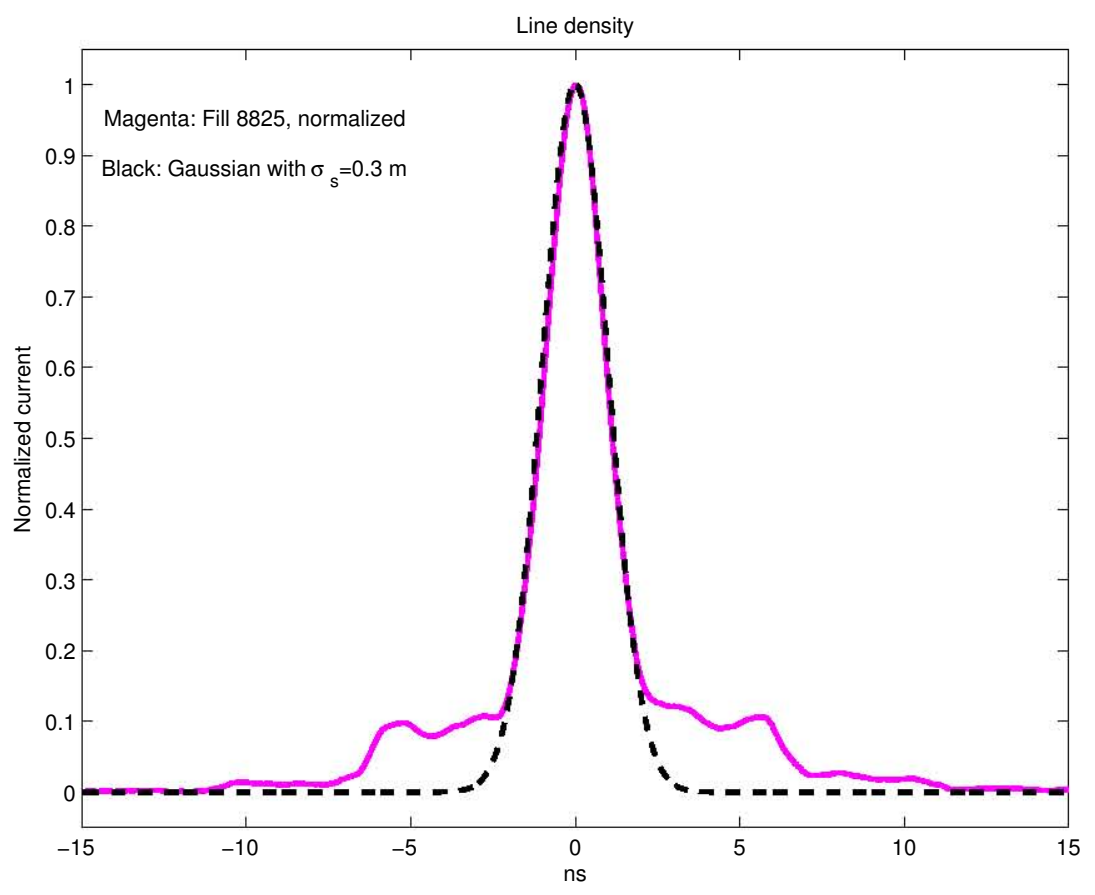

Figure 2: A Gaussian line density with $\sigma_{s}=0.3 \mathrm{~m}$ fits with the bunches after the rebucketing, Fill 8825, in run 2007.

\subsection{Luminosity}

The luminosity is defined as

$$
\mathcal{L}=\frac{M N_{y} N_{b} \beta \gamma f_{0}}{4 \pi \beta^{*} \epsilon_{N, r m s}}
$$

where $M$ is the bunch number, $N_{y}$ and $N_{b}$ are the bunch intensity of Yellow and Blue beams, respectively, $f_{0}$ is the revolution frequency, $\beta^{*}$ is the beta function at the IP, and $\epsilon_{N, r m s}$ is the normalized rms transverse emittance. The beams are assumed to be round and to have same size for both beams at the collision.

In RHIC, the luminosity is monitored by the Zero Degree Calorimeter (ZDC), which is installed at the forward direction of each side for all experiments. The $\mathrm{ZDC}$ rate, $r_{z d c}$, is related to the luminosity by

$$
r_{z d c}=\sigma_{z d c} \mathcal{L}
$$

where $\sigma_{z d c}$ is the cross section responsible for the ZDC coincidence rate.

\subsection{Collision}

With gold ion collisions, the nuclear and the mutual Coulomb dissociation cross sections are responsible for the coincidence rate detected by the ZDC. Additional 
beam loss comes from the Coulomb induced bound-electron-positron (pair) production, with $e^{-}$capture, and the single Coulomb dissociation as well.

For fully stripped gold ions colliding at $100 \mathrm{GeV} / n$, these cross sections are $[11,12]$,

\begin{tabular}{cc}
\hline \hline Cross section & barn \\
\hline Nuclear & 7.1 \\
Mutual Coulomb dissociation & 3.9 \\
Single Coulomb dissociation & 95 \\
Pair production with $e^{-}$capture & 117 \\
\hline ZDC, $\sigma_{z d c}$ & 11 \\
Total, $\sigma_{t o t}$ & 223 \\
\hline \hline
\end{tabular}

Table 3: Cross sections for fully stripped gold ions colliding at $100 \mathrm{GeV} / \mathrm{n}$

From RHIC gold runs starting from year 2000, the vernier scan performed for each run has measured the ZDC cross section always close to 11 barn [13].

From the best beam decay in store, the beam intensity loss rate is almost completely due to the burn-off, i.e., with the ratio of $\sigma_{t o t} / \sigma_{z d c} \approx 20$ from the coincident collisions. This has been observed many times in several runs.

Therefore, the ZDC and the total cross sections shown in Table 3 are good to use in the model.

\subsection{Intrabeam scattering}

The intrabeam scattering (IBS) significantly affect beam lifetime and emittance in RHIC heavy-ion operations.

For high energy beam, the longitudinal growth rate due to IBS can be simplified as [14-16],

$$
\tau_{\|}^{-1}=\frac{1}{\delta_{p}^{2}} \frac{d \delta_{p}^{2}}{d t} \approx \frac{r_{i}^{2} c N_{b h} \Lambda}{8 \beta^{3 / 2} \gamma^{3 / 2} \epsilon_{N, r m s}^{3 / 2}\left\langle\beta_{x}^{1 / 2}\right\rangle \sigma_{s} \delta_{p}^{2}}
$$

where $r_{i}$ is the classical radius of ion, $\delta_{p}$ is the rms momentum spread, $N_{b h}$ is the number of ions in a bunch, $\Lambda$ is the Coulomb logarithm, $\sigma_{s}$ is the rms bunch length, $\beta_{x}=\beta_{y}$ is the beta function, \langle\rangle denotes the average around the ring, and $\epsilon_{N, r m s}$ is the normalized rms transverse emittance.

The classical radius of gold ion is $r_{i}=48.6 \times 10^{-18} \mathrm{~m}$, and the Coulomb logarithm is usually taken as $\Lambda=10$. For the lattice used in RHIC run 2010, Au104, we have $\left\langle\beta_{x}^{1 / 2}\right\rangle \approx 6.93 \sqrt{m}$. Using other parameters listed in Table 1 , such as $N_{b h}=1.15 \times 10^{9}$ and $\epsilon_{N, r m s}=2.5 \pi \mu m$, we get the longitudinal growth rate at the early store as $\tau_{\|}^{-1}=2.5 \times 10^{-4}$, i.e., the longitudinal rise time is 1.11 hour. With a $30 \%$ intensity 
reduction to $N_{b h}=0.8 \times 10^{9}$ and a $30 \%$ emittance growth to $\epsilon_{N, r m s}=3.3 \pi \mu m$, the rise time would be 2.15 hour, given other parameters unchanged.

The transverse emittance growth rate due to the IBS can be approximated as,

$$
\tau_{\perp}^{-1}=\frac{1}{\epsilon_{N, r m s}} \frac{d \epsilon_{N, r m s}}{d t} \approx \frac{\gamma \delta_{p}^{2}}{\epsilon_{N, r m s}}\left\langle\frac{D_{x}^{2}+\left(\beta_{x} D_{x}^{\prime}+\alpha_{x} D_{x}\right)^{2}}{\beta_{x}}\right\rangle \tau_{\|}^{-1}
$$

where $D_{x}$ and $D_{x}^{\prime}$ are the dispersion function and its derivative with respect to $s$, $\alpha_{x}$ is the alpha function.

Here the H-function defined by

$$
H=\left\langle\frac{D_{x}^{2}+\left(\beta_{x} D_{x}^{\prime}+\alpha_{x} D_{x}\right)^{2}}{\beta_{x}}\right\rangle
$$

is an important factor in the transverse emittance growth.

For example, in RHIC run 2007, a lattice with 82 degree phase advance per FODO cell, Au72, is used. In run 2010, an IBS suppression lattice with 92 degree phase advance is used, which is Au104. The H-function of Au104 is about 15\% smaller than Au72, and hence, while the longitudinal growth rate is comparable, the transverse emittance growth rate of Au104 is smaller than Au72 by about $15 \%$ [17].

In specific, the transverse emittance rise time, with the beam parameters in Table 1, is 1.16 hour and 0.99 hour for Au104 and Au72, respectively.

\subsection{Hour-glass and other effects}

With zero crossing angle, and for Gaussian beam, the hour-glass factor can be approximated from the bunch length and the $\beta^{*}$ by [18],

$$
H_{G}(r)=\sqrt{\pi} r e^{r^{2}}(1-\operatorname{erf}(r))
$$

where $r=\beta^{*} / \sigma_{s}$ and $\operatorname{erf}(r)$ is the error function of $r$. For $\beta^{*}=0.7 m$ and $\sigma_{s}=0.3$ $m$ in Table $1, H_{G}=0.926$.

In RHIC, more factors associated with the luminosity calibration need to be considered.

- With some particles captured in the side buckets, the bunch shape is not perfect Gaussian, as shown in Fig.2.

- Two beams at the collision may not have the same size, and the beam may not be round. Also, in fact, the $\beta$ function measured at the IP is usually different in $x$ and $y$ directions [19].

- Limited ZDC detector acceptance and the high voltage (HV) applied to the detectors may also affect the ZDC coincidence rate. For example, in run 2008, a $3.5 \% \mathrm{HV}$ change to STAR west affected the ZDC rate by $15 \%$. In addition, the accidental coincidence requires corrections. 

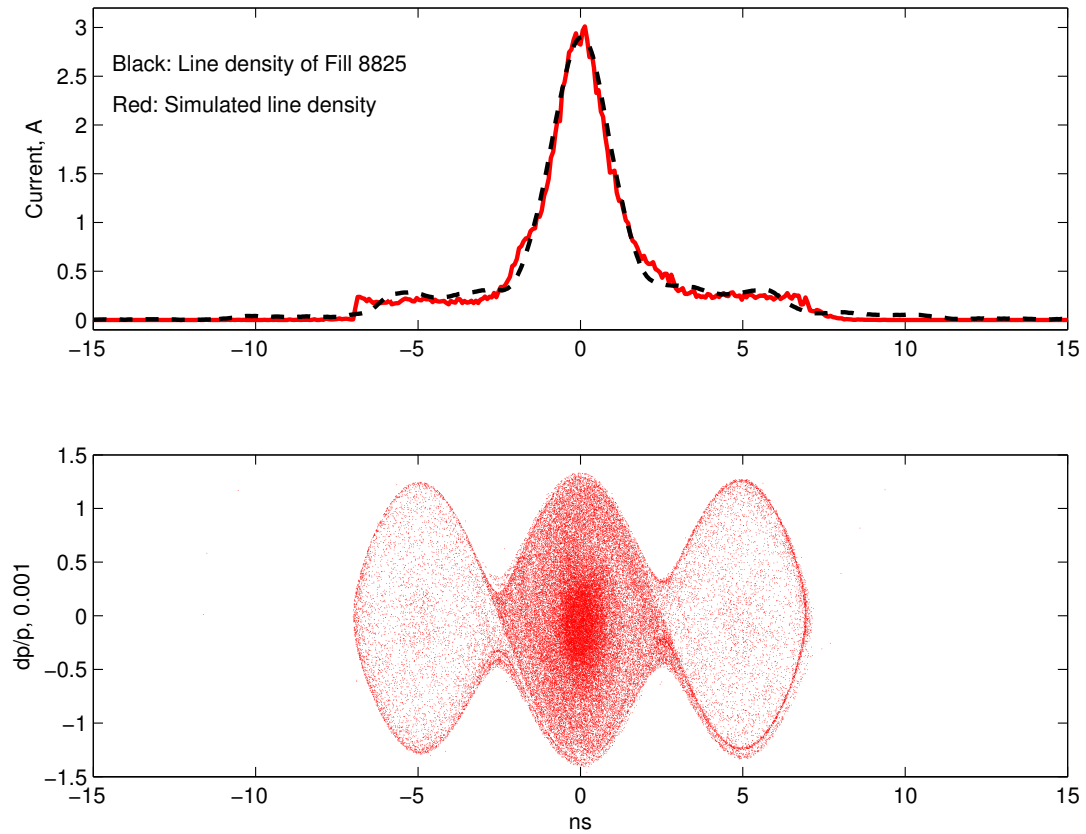

Figure 3: Phase space simulation of RHIC gold beam rebucketing with $300 \mathrm{kV}$ of $28 \mathrm{MHz}$ cavity and $3 \mathrm{MV}$ of $197 \mathrm{MHz}$ cavity in phase, the line density is compared with Fill 8825 in run 2007. Bunch intensity is $10^{9}$ ions.

In the model, therefore, the calculated hour-glass factor is added by another factor, which is subjected to modify but intended to keep as a constant.

\subsection{Beam loss}

It has been observed that the beam intensity reduction, in addition to the burn-off, can be mainly due to the longitudinal dynamics, i.e., the ions leak out of the RF bucket, and eventually get lost [20].

Firstly, note that the longitudinal IBS growth rate, $\tau_{\|}^{-1}$, is actually defined for the longitudinal emittance growth, i.e., $\epsilon_{\ell, 95 \%} \propto \delta_{p}^{2}$. This is because,

$$
\epsilon_{\ell, 95 \%}=\delta_{p}^{2} \sqrt{\frac{8 \pi|\eta| \gamma^{3} E_{0}^{3}}{e h V f_{0}^{2}}}
$$

where $f_{0}$ is the revolution frequency, and $\eta$ is the slippage factor. All parameters on the right side of the equation are constant at the beam store, except the momentum spread, $\delta_{p}$. Therefore, the longitudinal IBS rise time can be used for the longitudinal emittance growth rate, which leads to the beam loss.

In Fig.3, the simulated particle distribution in the longitudinal phase space and the line density are shown. This is compared with the measured beam line density of Fill 8825, where the $28 \mathrm{MHz}$ cavity with $300 \mathrm{kV}$, and $197 \mathrm{MHz}$ cavity with $3 \mathrm{MV}$ are used in the rebucketing. The bunch intensity is $10^{9}$ ions. 
In the early store, with the fast longitudinal emittance growth, the ions in the central bucket would gradually move to side buckets. These particles are still detected by the DC transformer, and still engaged in the ZDC collisions. Only in the later, with larger longitudinal emittance growth, beam loss starts to take place. After examining the fills in 2007 run, the beam loss is modeled not only by the IBS longitudinal emittance growth rate, $\tau_{\|}^{-1}$, but also by a factor of $f=0.2 \times t^{0.6}$, where $t$ is the time from the beginning of the collision. The factor $f$ is set and locked in the model.

\subsection{The model}

In the tracking, we assume that the two beams have same size, and also the beams are round. Total 6 dynamic variables are used.

$$
\begin{aligned}
\frac{d \epsilon_{T b, T y}}{d t} & =\frac{\epsilon_{T b, T y}}{\tau_{I B S, T}\left(N_{b, y}, \epsilon_{\ell b, \ell y}, \epsilon_{T b, T y}\right)}-\frac{\epsilon_{T b, T y}}{\tau_{c o o l, T}\left(N_{b, y}, \epsilon_{\ell b, \ell y}, \epsilon_{T b, T y}\right)} \\
\frac{d \epsilon_{\ell b, \ell y}}{d t} & =\frac{\epsilon_{\ell b, \ell y}}{\tau_{I B S, \ell}\left(N_{b, y}, \epsilon_{\ell b, \ell y}, \epsilon_{T b, T y}\right)}-\frac{\epsilon_{\ell b, \ell y}}{\tau_{c o o l, \ell}\left(N_{b, y}, \epsilon_{\ell b, \ell y}, \epsilon_{T b, T y}\right)} \\
\frac{d N_{b, y}}{d t} & =-\frac{N_{b, y}}{\tau_{I B S, N}\left(N_{b, y}, \epsilon_{\ell b, \ell y}, \epsilon_{T b, T y}\right)}-\frac{N_{b, y}}{\tau_{\mathcal{L}}\left(N_{b, y}, \epsilon_{\ell b, \ell y}, \epsilon_{T b, T y}\right)}
\end{aligned}
$$

Here $\epsilon_{T b, T y}$ and $\epsilon_{\ell b, \ell y}$ are the transverse and longitudinal emittance of Blue and Yellow beams, respectively. $\tau_{I B S, T}$ and $\tau_{I B S, \ell}$ are the instantaneous IBS rise time, $\tau_{I B S, N}$ is the one discussed in the subsection of beam loss. $\tau_{\text {cool }, T}$ and $\tau_{\text {cool, } \ell}$ are the instantaneous cooling time, and $\tau_{\mathcal{L}}$ is the instantaneous lifetime from burn-off.

The ZDC collision rate, the luminosity, and the integrated luminosity are also produced in the tracking.

\section{Comparison of RHIC fills with the model}

In this section, the model is used to compare with Fill 8908 in run 2007 without cooling, and Fill 8825 with Yellow longitudinal cooling. Based on this, a comparison of the beam intensity, the transverse emittance, and the luminosity of 3 fills with same initial condition, but the first without cooling, the second with one beam longitudinal cooling, and the third with longitudinal cooling of both beams, are made. 

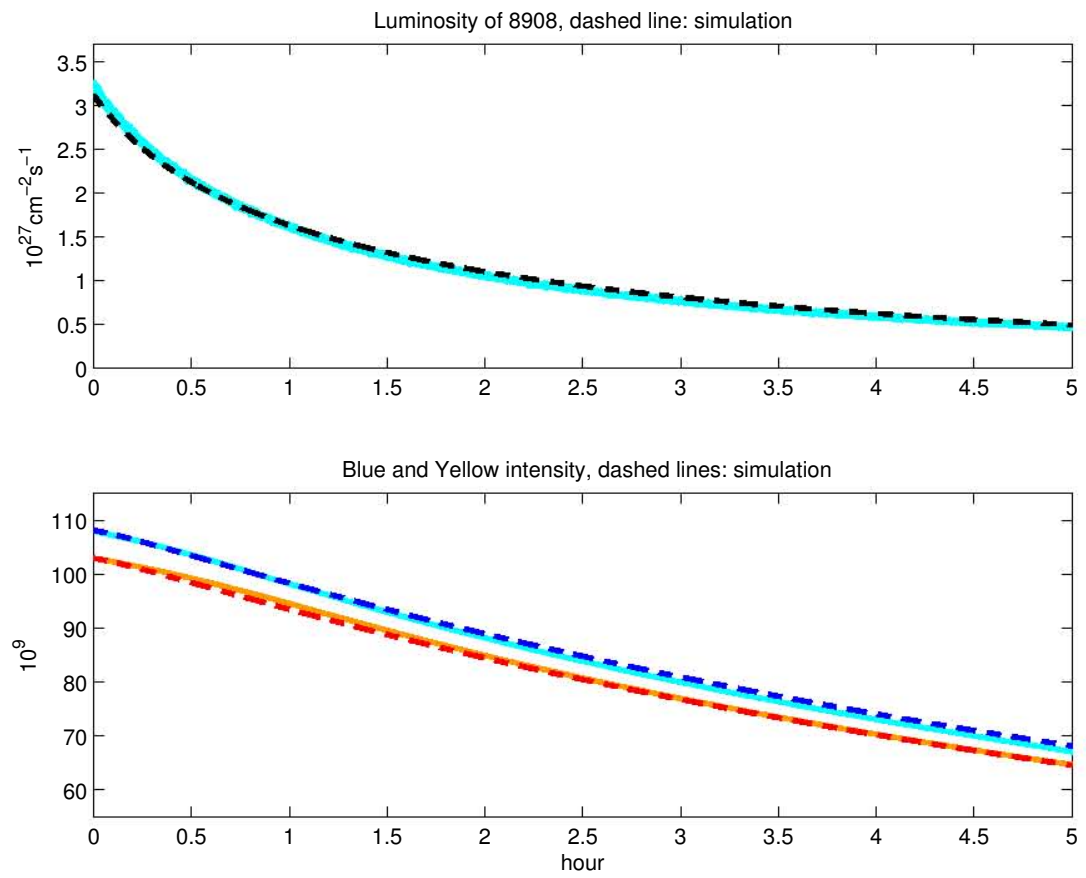

Figure 4: The Blue and Yellow intensity, and the luminosity of Fill 8908, which is without cooling, compared to the simulation with the luminosity model, for 5 hours.

\section{$5.1 \quad$ Fills 8908 and 8825}

Fills 8908 and 8825 are both good physics fills in run 2007, but not necessarily the best ones. Fill 8908 has no cooling, while Fill 8825 has a longitudinal stochastic cooling applied to the Yellow beam. Parameters of 8908 and 8825 are shown in Table 4.

\begin{tabular}{cccc}
\hline \hline Parameter & Unit & 8908 & 8825 \\
\hline$\beta^{*}$ & $m$ & 0.8 & 0.8 \\
Bunch number & & 103 & 103 \\
Blue intensity & $10^{9}$ & 108.3 & 106.1 \\
Yellow intensity & $10^{9}$ & 103.1 & 105.1 \\
Normalized rms emittance & $\pi \mu m$ & 2.69 & 2.82 \\
PHENIX ZDC rate & $10^{3}$ & 36.7 & 35.1 \\
Peak luminosity & $10^{27} \mathrm{~cm}^{-2} \mathrm{~s}^{-1}$ & 3.34 & 3.19 \\
Yellow long. cooling & & no & yes \\
Blue long. cooling & & no & no \\
\hline \hline
\end{tabular}

Table 4: Fill 8908 and Fill 8825, the beam parameters are at the early collision 

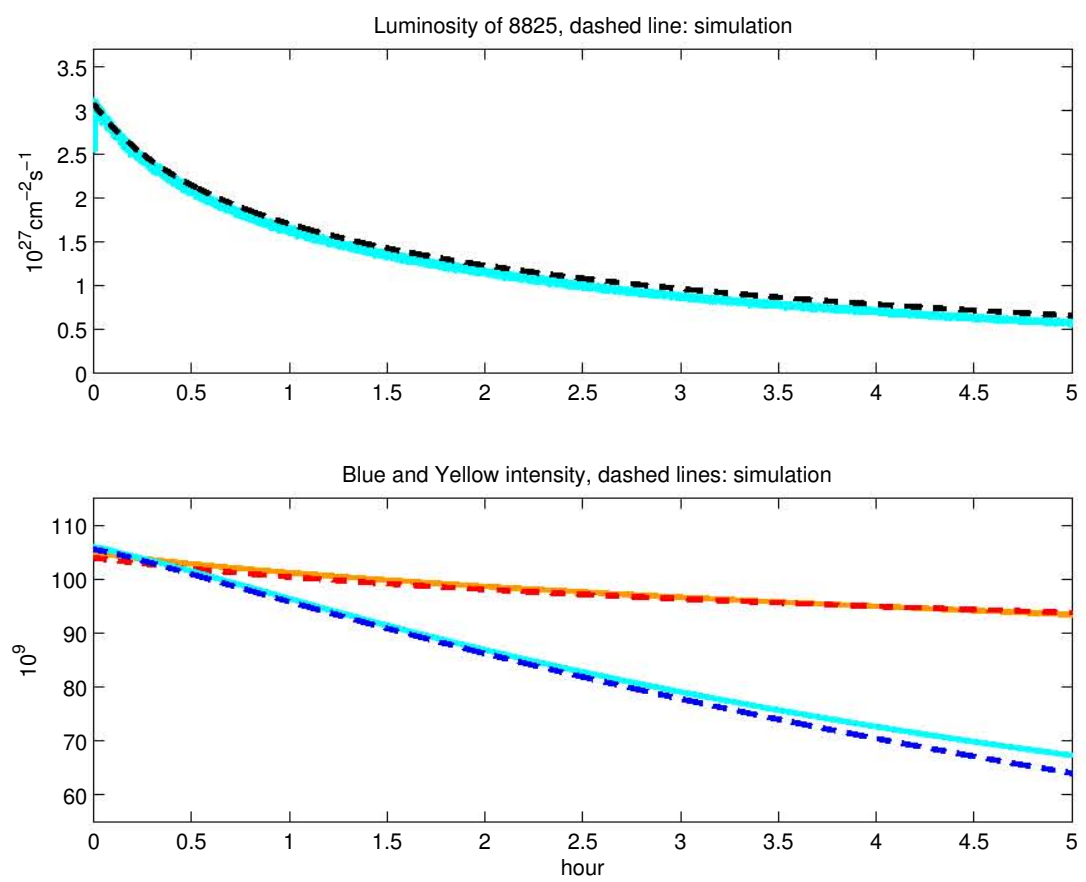

Figure 5: The Blue and Yellow intensity, and the luminosity of Fill 8825, which has longitudinal cooling for Yellow beam, compared to the simulation with the luminosity model, for 5 hours.

The initial conditions at the early store are similar, but not identical for Fill 8908 and 8825. In the tracking, the initial intensities are taken from Table 4, but the initial transverse emittance, the longitudinal emittance, the bunch length, and the momentum spread are set the same, as that in Table 1. Also, all other parameters, such as the hour-glass factor, the beam loss correction factor, the cross sections of the collision, and the lattice related parameters, are the same for these two fills.

In Fig.4, the simulation is compared with Fill 8908 for the time evolution of the Blue and Yellow intensities, and the luminosity as well.

In Fig.5, the simulation is compared with Fill 8825 for the Blue and Yellow intensities, and the luminosity as well.

Note that the transverse emittance of Fill 8908 and Fill 8825 are slightly different from what is used in the model, $2.5 \pi \mu \mathrm{m}$, and the longitudinal emittance is also different from that in the model, $0.7 \mathrm{eVs} / \mathrm{n}$. This leads to some discrepancy between the simulation and the measured parameters.

Otherwise, the simulation is close enough to the real fills in run 2007, both for that without cooling, and that with longitudinal cooling of one beam. 

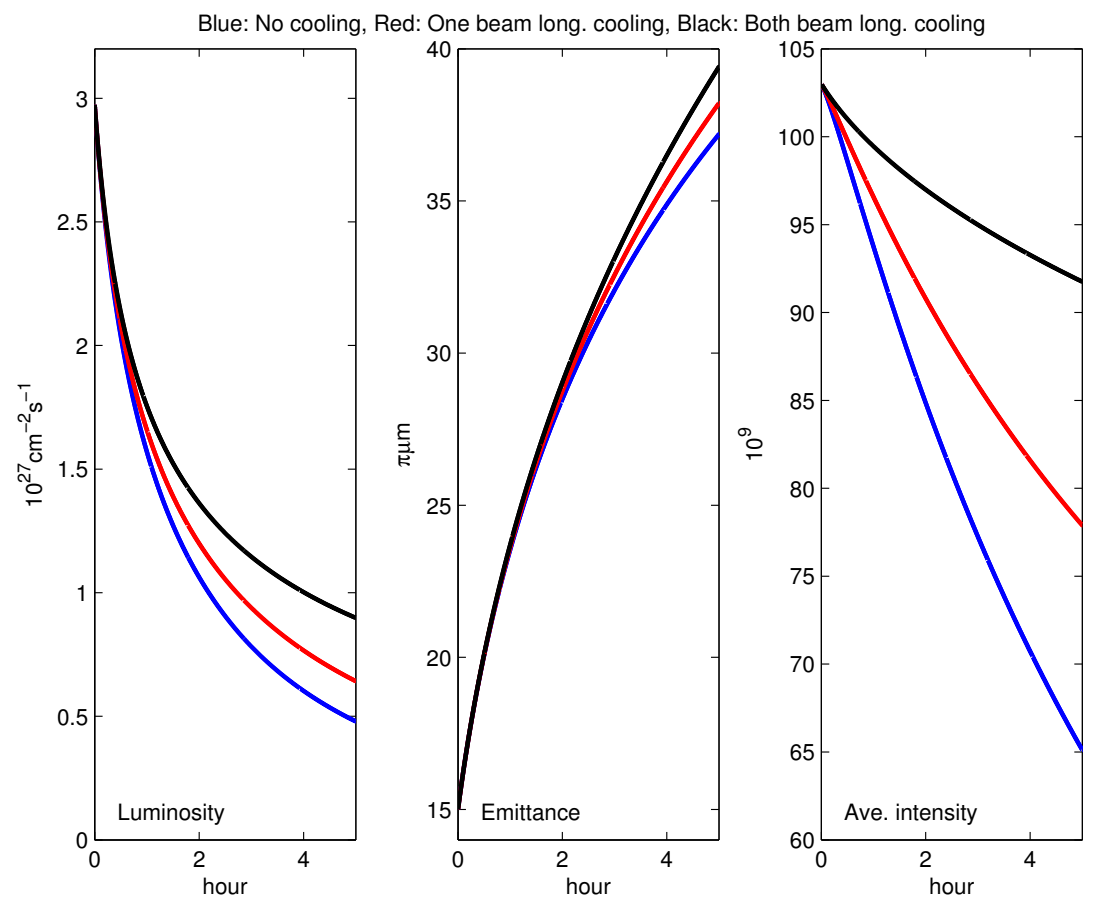

Figure 6: The beam intensity, the transverse emittance, both are average of the Blue and the Yellow, and the luminosity of fills with same initial parameters, but different longitudinal cooling: without, with one, and with two beams

\subsection{A fill with two coolings}

With the good comparison of the tracking with Fill 8908 and Fill 8825, a fill with the longitudinal cooling applied to both beams can be projected. The gain of the luminosity with different longitudinal coolings can be compared.

\begin{tabular}{ccccc}
\hline \hline Parameter & Unit & Fill 01 & Fill 02 & Fill 03 \\
\hline$\beta^{*}$ & $m$ & 0.8 & 0.8 & 0.8 \\
Bunch number & & 103 & 103 & 103 \\
Blue intensity & $10^{9}$ & 103 & 103 & 103 \\
Yellow intensity & $10^{9}$ & 103 & 103 & 103 \\
Normalized rms emittance & $\pi \mu m$ & 2.5 & 2.5 & 2.5 \\
Peak luminosity & $10^{27} \mathrm{~cm}^{-2} \mathrm{~s}^{-1}$ & 2.97 & 2.97 & 2.97 \\
Yellow long. cooling & & no & yes & yes \\
Blue long. cooling & & no & no & yes \\
\hline \hline
\end{tabular}

Table 5: Parameters for comparison of fills without cooling, with longitudinal cooling of one beam, and with longitudinal cooling of both beams 
Let the modified Fill 8908, renamed Fill 001, and the modified Fill 8825, renamed Fill 002, to have the same conditions as the new one, Fill 003. Parameters of these 3 fills are shown in Table 5.

In Fig.6, the beam intensity, the transverse emittance, both are average of the Blue and the Yellow, and the luminosity, are shown for the fills having the same initial condition, but with different longitudinal coolings. In Table 6, the Blue and Yellow averaged beam intensity, the transverse emittance, and the luminosity at the end of store, in 5 hours, are shown. The integrated luminosity are also shown. Compared with the fill without cooling, the integrated luminosity is increased by $11.6 \%$ if one beam has the longitudinal cooling, and $26.6 \%$ if both beams are cooled.

\begin{tabular}{ccccc}
\hline \hline Parameter & Unit & 001 & 002 & 003 \\
\hline Beam intensity & $10^{9}$ & 65.1 & 77.9 & 91.8 \\
Normalized rms emittance & $\pi \mu m$ & 6.20 & 6.37 & 6.57 \\
Luminosity & $10^{27} \mathrm{~cm}^{-2} \mathrm{~s}^{-1}$ & 0.48 & 0.64 & 0.90 \\
Integrated luminosity & $\mu b^{-1}$ & 19.9 & 22.2 & 25.2 \\
Yellow long. cooling & & no & yes & yes \\
Blue long. cooling & & no & no & yes \\
\hline \hline
\end{tabular}

Table 6: Fills without cooling, with longitudinal cooling applied to one beam , and to both beams. The beam intensity, the transverse emittance, both averaged for Blue and Yellow, and the luminosity at the end of store, 5 hours. The integrated luminosity of the store are also shown.

\section{Model development}

Further model development depends on the need of the RHIC gold run.

- With the transverse stochastic cooling applied on one beam (such as that in run 2010), two beams collide with different sizes. This needs to be modelled, but only a first-order approximation is considered. When the transverse emittance of beam one is reduced, but not the beam two, two things happen.

1. The first is that the particle density in beam one is enhanced, and the collision probability is increased if other parameters keep unchanged. This is simply modeled using the reduced emittance of beam one.

2. The second is that the number of particles in beam two engaging in collision is reduced. Let the distribution be Gaussian in both directions, $\rho(x, y)$, and let the cut be $x=y= \pm k \sigma$, where $\sigma$ is the standard deviation, then we have

$$
\iint_{-k \sigma}^{k \sigma} \rho(x, y) d x d y=1-e^{-k}
$$


and this relation will be used for the modeling.

- In run 2010, with only vertical cooling and by the beam coupling, the beam transverse emittance can be reduced by half in less than 1 hour. This implies that the beam-beam effect may need to be considered. The effect of the beam-beam on the proton runs in RHIC has been observed and studied [7]. What learned there can be used as a first try in the luminosity model. Better approach may be obtained once the gold run is achieving larger beam-beam effect.

- A challenge in run 2010 is that when the transverse cooling is applied to one beam, the intensity lifetime of the other beam is affected. This effect is not trivial. Another, bigger, challenge is the large beam loss at the early store, which has a pattern that it is gradually reduced and then disappeared in about two hours. Once a better understanding of the mechanism becomes available, the model would follow.

- With both the longitudinal and transverse coolings applied, the store time should be extended, then the optimized store time is a question. Several other questions also arise. These include how to choose the optimized $\beta^{*}$, the optimized bunch intensity, and the optimized transverse emittance.

\section{Acknowledgment}

I would like to thank M. Bai and M. Blasckewicz for suggesting this work.

\section{References}

[1] http://www.agsrhichome.bnl.gov/RHIC/Runs/

[2] W. Fischer, Proceedings of IPAC10, p.1227, Kyoyo, Japan, 2010

[3] M. Blaskiewicz, J.M. Brennan, and K. Mernick, PRL, 105, 094801, 2010

[4] S.Y. Zhang and V. Ptitsyn, PRST AB, 11, 051001, 2008

[5] M.J. Rhoades-Brown and M.A. Harrison, AD/RHIC-106, BNL, 1991

[6] D. Trbojevic, et.al., Proceedings of EPAC, p.1416, Paris, France, 2002

[7] S.Y. Zhang and V. Ptitsyn, Proceedings of PAC07, p.1886, Albuquerque, New Mexico, 2007

[8] R. Bruce, J.M. Jowett, M. Blaskiewicz, and W. Fischer, PRST AB, 13, 091001, 2010 
[9] Y. Luo, X. Gu, W. Fischer, D. Trbojevic, C-A AP 419, BNL, Jan. 2011

[10] S.Y. Zhang, C-A AP 420, BNL, Jan., 2011

[11] A.J. Baltz, M.J. Rhoades-Brown and J. Weneser, PR E54, 4233, 1996

[12] A.J. Baltz, C. Chasman, and S.N. White, NIM, A 417, 1-8, 1998

[13] A. Drees, private communication

[14] A. Sorenson, CERN-87-10, p.135, 1987

[15] A. Piwinski, Int. Conf. High Energy, Stanford, 1974

[16] A.V. Fedotov, C-A AP 168, BNL, Sep., 2004

[17] A.V. Fedotov, et. al., Proceedings of Hadron Beam, p.148, Nashville, Tennessee, 2008

[18] H. Burkhardt and P. Grafstron, LHC Project report, 1019, CERN, 2007

[19] M. Bai, private communication

[20] S.Y. Zhang, C-A AP, in preparation, 2011 